\title{
ENSINO E ORIENTAÇÃO PARA PROJETO DE CONVERSORES CC-CC ATRAVÉS DE APLICATIVOS EM JAVA
}

\author{
Carlos Alberto Canesin*
}

\author{
Flávio Alessandro Serrão Gonçalves*
}

\author{
Leonardo Poltronieri Sampaio* \\ *Laboratório de Eletrônica de Potência - DEE-FEIS-UNESP \\ Av. Brasil, 56 \\ CEP 15385-000 - Ilha Solteira (SP)
}

\section{RESUMO}

Este trabalho apresenta um novo conjunto de ferramentas de simulação interativas abordando a operação idealizada de conversores CC-CC não isolados, visando o auxílio e aprimoramento do ensino em Eletrônica de Potência. Estas novas ferramentas de simulação utilizam uma metodologia consolidada de desenvolvimento, empregando recursos modernos da linguagem Java, para propiciar um ambiente dinâmico e interativo de visualização de simulações em regime permanente. Adicionalmente, este trabalho apresenta as principais características operacionais das ferramentas educacionais propostas, e, aborda sua utilização como ferramenta de projeto orientado para as aulas de laboratórios no curso de Eletrônica de Potência. Neste sentido, com o objetivo de validar as ferramentas desenvolvidas e mostrar a sua utilidade, alguns exemplos considerando o conversor Buck são apresentados, onde são efetuadas comparações com ambientes de simulação conhecidos, e com resultados experimentais obtidos nos laboratórios didáticos.

PALAVRAS-CHAVE: Ferramentas educacionais, Métodos educacionais, Simulação de conversores CC-CC, Projetos de conversores CC-CC.

Artigo submetido em 18/03/2009 (Id.: 00975)

Revisado em 22/05/2009, 24/09/2009, 16/10/2009, 06/11/2009

Aceito sob recomendação do Editor Associado Prof. Enes Gonçalves Marra

\begin{abstract}
Education and Oriented Design for DC to DC Convertes Using Java Applets Tools

This paper presents a new set of interactive simulation tools dealing with the steady-state operation of idealized nonisolated DC to DC converters, in order to support the enhancement of power electronics education. These new interactive tools make use of a consolidated design approach where the benefits of Java language were utilized to provide a dynamic and interactive visualization of simulations in steady-state. Additionally, this paper discusses the main features of proposed simulation tools, and discusses its use as an oriented design tool for the lectures and laboratory experiments in the Power Electronics courses. In order to corroborate the proposed interactive simulation tools and to show its usefulness, comparisons of results from Buck converter on proposed tools and a well-known simulator package with those on experimental evaluation from laboratory classes are presented.
\end{abstract}

KEYWORDS: Educational Tool, Education Methodology, DC-DC converter simulation, DC-DC converter design.

\section{INTRODUÇÃO}

O aprendizado ou ensino de um tópico em engenharia, principalmente na área de eletrônica de potência, muitas vezes é complicado porque existe um grande número de reações pos- 
síveis de um sistema quando ocorrem alterações em seus parâmetros de operação. Desta forma, os comportamentos dinâmicos de um sistema podem ser demonstrados facilmente em muitos casos se estes comportamentos forem apresentados através de um ambiente de treinamento/ensino interativo. Além disso, este procedimento evita que o estudante atue de forma passiva durante o aprendizado, tornando o aluno ativo durante o processo educacional (Hwang, 1998; Agelidis, 2005).

Por outro lado, é muito importante prover ao estudante as experiências práticas através dos experimentos nas aulas de laboratório. As experiências práticas propiciam ao estudante o senso de realidade, onde podem visualizar claramente alguns fenômenos importantes na operação de circuitos e componentes (recuperação reversa do diodo, perdas/esforços nos processos de comutação, entre outras não idealidades), e ainda adquirir conhecimentos/habilidades no manuseio de equipamentos (osciloscópios e equipamentos de medição).

Neste sentido, a evolução nos computadores associada com a rápida expansão da internet e suas ferramentas forneceram aos educadores um mecanismo poderoso e eficaz de entrega de informações. Este ambiente pode facilmente incorporar as mais recentes tecnologias multimídia (simulações interativas, vídeos/sons, banco de dados, equipamentos controlados remotamente), permitindo a criação e o gerenciamento de cursos educacionais, laboratórios virtuais e salas de aula virtuais (Drofenik e Kolar, 2002; Oliveira Júnior e Silva, 2004; Harb et alii, 2005; Bartal et alii, 2005; Silva e Cunha, 2006; Garcia et alii, 2008).

Nos últimos anos, muitos pesquisadores estão aderindo ao conceito de laboratórios virtuais, onde os experimentos são totalmente executados de maneira remota. As principais vantagens apresentadas por estas metodologias são a independência de tempo e espaço, a operação através de uma interface simples e familiar, exigindo apenas algumas configurações de parâmetros, e, a possibilidade de conexões simultâneas, considerando os modos ativo e passivo.

No modo ativo o usuário atua efetivamente na realização do experimento, enquanto no modo passivo, devido a restrições do sistema, o usuário passivo atua apenas no papel de expectador de outros usuários ativos.

No entanto, os estudantes de certa maneira ainda continuam atuando de forma passiva. Devido à similaridade de ambientes os ensaios experimentais remotos podem ser entendidos como se fossem uma simulação, e o senso prático de realidade e das fronteiras de operação podem ser mal interpretados (Bauer et alii, 2008; Wu et alii, 2008; Pleskacz et alii, 2008).

Além disso, os alunos podem aproveitar as vantagens da ex- periência real e da simulação virtual de forma simultânea se as atividades de laboratório forem complementadas com simulações interativas (Gonçalves e Canesin, 2002; Gonçalves e Canesin, 2005; Donoso-Garcia et alli, 2008).

Neste contexto, considerando a evolução da metodologia de desenvolvimento consolidada em (Canesin et alii, 2008), onde foram apresentados os retificadores CA-CC, este artigo apresenta novas ferramentas de simulação interativas abordando os principais aspectos dos conversores CC-CC nãoisolados e em regime permanente. Basicamente, as ferramentas abordam os tópicos relacionados com o projeto, as etapas de operação, os modos de condução, os caminhos do fluxo de corrente, e as formas de onda nos componentes, além dos esforços de tensão e corrente. As novas ferramentas podem ser utilizadas tanto nas aulas convencionais, quanto nas aulas práticas de laboratório.

\section{ESTRUTURA DAS FERRAMENTAS DE SIMULAÇÃO INTERATIVA DOS CON- VERSORES CC-CC}

O objetivo principal das ferramentas de simulação propostas é produzir um ambiente interativo de simulações idealizadas para conversores $\mathrm{CC}-\mathrm{CC}$ não-isolados em regime permanente.

Neste sentido, o leiaute visual e o comportamento da interface gráfica do usuário (GUI - Graphic User Interface) são partes importantes do desenvolvimento de um software.

A elaboração da interface de comunicação entre o usuário e a ferramenta de simulação deve assegurar que a linguagem visual introduzida na GUI seja adequada às tarefas que deve executar para propiciar elevado grau de interatividade e facilidade de uso.

A interface gráfica proposta para as ferramentas de simulação foi desenvolvida empregando quatro áreas distintas, como mostra a Figura 1, e tem o propósito de permitir que o estudante através de componentes visuais modifique os parâmetros do circuito, execute as simulações e visualize os resultados de maneira direta e fácil.

As áreas estão interconectadas e dividas em duas categorias: ativa e passiva. As áreas ativas permitem a interação com o estudante através da solicitação de informações sobre os parâmetros necessários no projeto do conversor. Enquanto as áreas passivas fornecem as informações resultantes por meio de formas de ondas e soluções de métodos numéricos.

A área 1, denominada ativa, permite ao estudante modificar os parâmetros do circuito carregados previamente com o projeto padrão do conversor (tensão de entrada e de saída, 


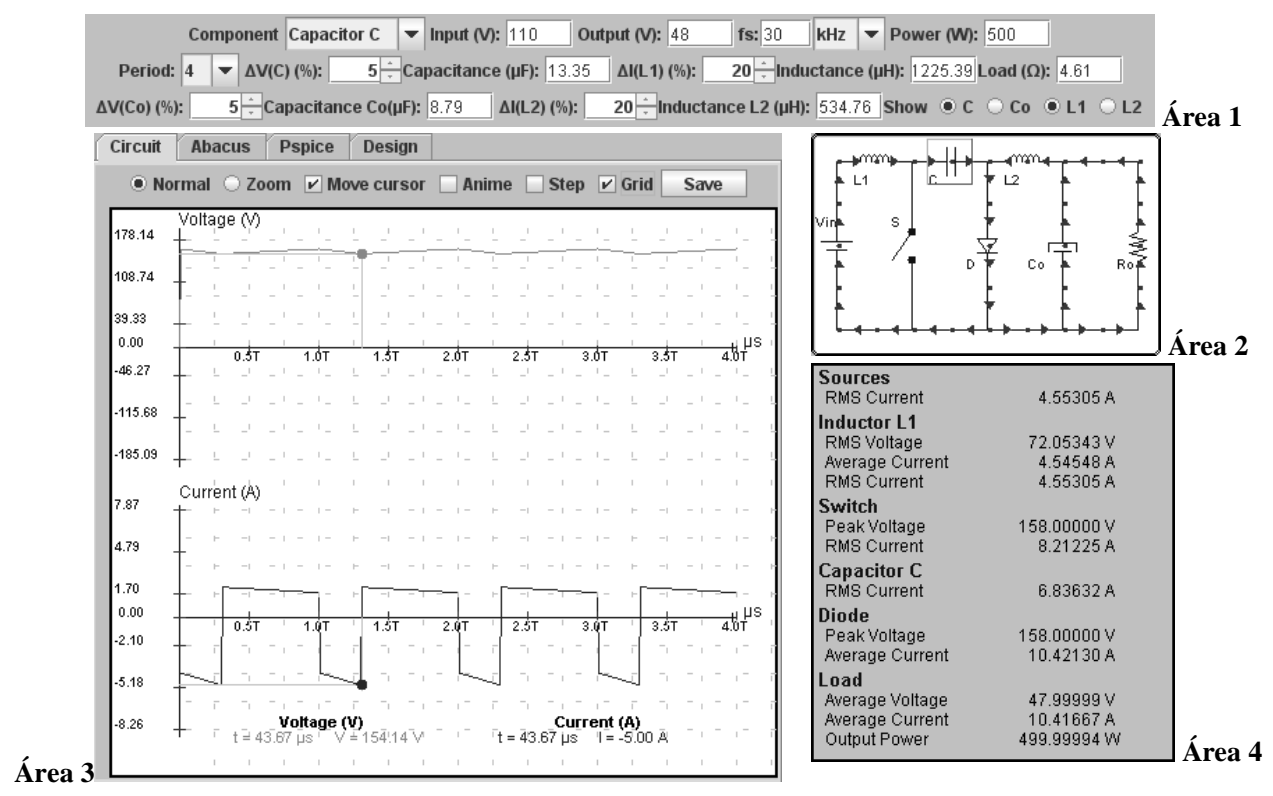

Figura 1: Interface gráfica do usuário desenvolvida para as ferramentas de simulação.

frequência de chaveamento, ondulações de corrente e de tensão). Adicionalmente, caso o estudante forneça dados inconsistentes para a ferramenta de simulação, serão enviados avisos pelos setores passivos informando as prováveis causas do erro.

Um módulo de desenho do circuito esquemático (MDCE), com uma estrutura baseada em uma matriz $5 \times 9$, constitui a área ativa 2. Basicamente, o circuito é criado considerando valores e atributos descritos nas linhas e colunas da matriz. Um banco de dados foi desenvolvido contendo a codificação de informações dos componentes e de leiaute (orientação e posição). Além disso, o módulo MDCE é interconectado com o módulo das formas de ondas (área 3), e, recebe deste a relação das etapas de operação para ilustrar na forma de animação o fluxo de corrente pelos ramos do circuito, mostrando inclusive os estados do interruptor (bloqueado ou conduzindo).

A área ativa 3 é composta por dois módulos gráficos. O primeiro módulo possui a finalidade de desenhar as formas de onda de tensão e corrente do componente selecionado no circuito da topologia presente na área ativa 2.

Um gráfico interativo de regiões de operação relacionado com a topologia do conversor analisado compõe o segundo módulo. O módulo desenha diversas curvas referentes aos planos de operação usando a relação do fator de carga $(\gamma)$ em função do ganho estático $\left(\mathrm{V}_{\text {saída }} / \mathrm{V}_{\text {entrada }}\right)$, e tomando a razão cíclica (D - Duty Cycle) como parâmetro discreto. Onde, o fator de carga $(\gamma)$ representa uma grandeza normalizada em função dos parâmetros do conversor, relacionada com a potência processada.

Dois cursores móveis estão disponíveis sobre as curvas do ábaco, permitindo que o ponto de operação possa ser modificado dinamicamente e de maneira imediata através do uso do mouse. Nesta ferramenta é possível alterar de forma interativa o modo de condução do conversor (contínua, descontínua e crítica), e visualizar tais alterações diretamente nas formas de onda de simulação.

Além disso, a área 3 disponibiliza um arquivo no formato compatível com o software de simulação Pspice referente ao ponto de operação avaliado na ferramenta, e uma área onde as equações empregadas na determinação do valor dos componentes são ilustradas.

A área 4, denominada passiva, possui a finalidade de exibição de informações. Esta recebe os dados referentes às formas de onda de tensão e corrente da área 3, e exibe os valores de pico, eficaz e médio empregando resultados do algoritmo matemático da série de Fourier, considerando até a trigésima componente harmônica nos cálculos.

\section{CLASSES BÁSICAS DE DESENVOLVI- MENTO DAS FERRAMENTAS DE SIMU- LAÇÃO}

As ferramentas de simulação foram desenvolvidas utilizando recursos modernos de programação providos pela linguagem Java, especificamente a técnica de orientação a objetos (OO). 


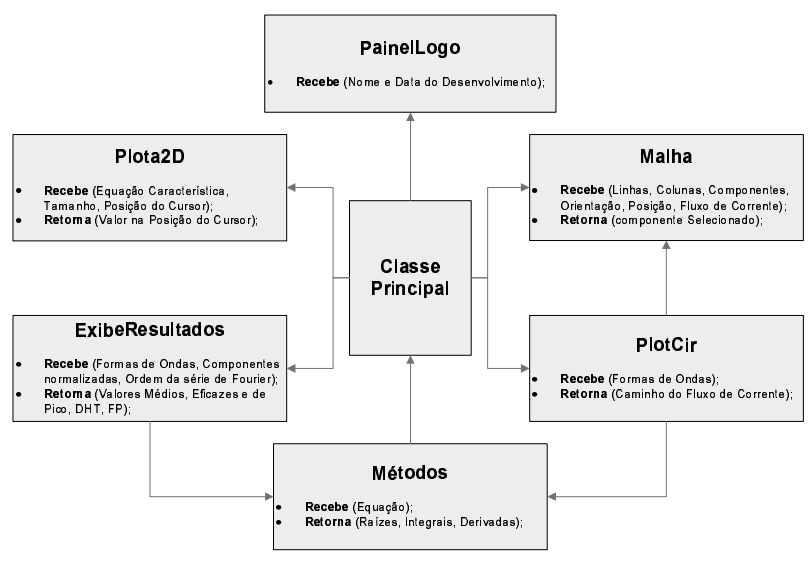

Figura 2: Estrutura mínima de classes requeridas para o desenvolvimento de uma nova ferramenta de simulação.

Assim, o processo de criação de um novo aplicativo, referente a uma nova estrutura de conversor, é baseado em um procedimento direto de incorporação e reutilização das classes primárias desenvolvidas para a composição da estrutura básica necessária ao aplicativo, conforme a Figura 2. As classes primárias se comunicam entre si através de matrizes paramétricas contendo dados, resultados dos algoritmos matemáticos e comandos de controle. Desta forma, a estrutura mínima de classes primárias requeridas para o desenvolvimento de uma nova ferramenta deve ser composta pelas seguintes classes:

Malha: classe responsável pelos desenhos dos circuitos esquemáticos;

PlotCir: classe responsável pelo desenho das formas de ondas;

ExibeResultados: classe responsável pela exibição das informações dos resultados dos cálculos numéricos;

Métodos: classe para implementação das rotinas dos métodos de cálculo numérico empregados;

PainelLogo: classe responsável pela identificação do aplicativo (conversor, versão, autores, etc);

Plota2D: classe responsável pelo desenho e interação com os ábacos matemáticos.

\section{FERRAMENTAS DE SIMULAÇÃO INTE- RATIVA DESENVOLVIDAS}

Atualmente, as simulações interativas disponíveis para conversores CC-CC não-isolados abrangem os conversores Buck, Boost, Buck-boost, Sepic, Zeta e Æuk (Barbi e Martins, 2000; Erickson e Maksimovic, 2002; Mohan et alii,
1995; Rashid e Rashid, 2006).

As ferramentas de simulação possibilitam a visualização e análise dos conversores CC-CC considerando os modos de condução contínua (MCC), descontínua (MCD) e na região crítica (MCCr).

As Figuras 3 a 6 ilustram uma série de exemplos mostrando os recursos disponíveis nas ferramentas de simulação. Os estudantes podem modificar os parâmetros do circuito e de operação, e avaliar os efeitos na operação do conversor analisado de forma "on-line" (principais variáveis de estado, formas de onda de tensão e corrente e o modo de operação do conversor).

A Figura 3 ilustra a ferramenta de simulação para o conversor Boost com a opção "Move Cursor" ativada, onde dois cursores móveis estão disponíveis nas formas de onda de tensão e de corrente. Estes cursores podem ser deslocados ao longo das formas de onda proporcionando ao estudante os valores instantâneos de tensão e corrente, e a visualização do fluxo de corrente no circuito esquemático, ilustrando as etapas de operação do conversor. Além disso, os cursores podem ser movimentados automaticamente ao longo das formas de onda mostrando as etapas de funcionamento se a opção "Anime" for ativada. Quando uma visualização mais detalhada das formas de onda é necessária existe uma opção de "zoom" disponível. Neste recurso pode ser desenhado um retângulo sobre a forma de onda de tensão, pelo aluno usando o mouse, e a área selecionada é redesenhada conforme a nova escala estabelecida (eixos horizontais e verticais).

Além disso, a visualização da forma de onda de corrente é ajustada apenas em seu eixo horizontal. Por outro lado, quando o retângulo é desenhado sobre a área de forma de onda de corrente, o processo ocorre de maneira análoga ao da forma de onda de tensão.

A área 4 utiliza os dados relacionados com as formas de onda de tensão e corrente exibidas na área 2 para calcular alguns índices importantes para as análises educacionais (valores eficaz, médio e de pico).

Para a obtenção de resultados numéricos com uma precisão adequada, estes índices são calculados usando o algoritmo de Fourier considerando trinta componentes harmônicas (Erickson e Maksimovic, 2002; Ramos et alli, 2006).

Um exemplo de interação com o módulo do ábaco de regiões de operação para o conversor Zeta é mostrado na Figura 4. A ferramenta desenha uma série de curvas mostrando a característica de carga normalizada $(\gamma)$ como uma função do ganho estático $\mathrm{G}$ (relação entre $\mathrm{V}_{\text {saída }}$ e $\mathrm{V}_{\text {entrada }}$ ) para o conversor Zeta, considerando diferentes valores de razão cíclica (D). Uma forma de onda referente à razão cíclica do 
ponto de operação analisado na simulação é mostrada na cor azul. Além disso, o módulo do ábaco desenha na cor verde a região de fronteira (MCCr) entre o módulo de condução contínua e descontínua, possibilitando uma melhor visualização entre os limites de operação.

O ponto de operação em regime permanente pode ser modificado através de dois cursores disponíveis na ferramenta do ábaco. O cursor azul possibilita a variação da razão cíclica (D), e o cursor vermelho permite a variação do ganho (G), de acordo com o fator de carga $(\gamma)$.

A Figura 5 mostra o exemplo da utilização do módulo denominado "Pspice" para ferramenta de simulação do conversor Sepic. O módulo tem a finalidade de proporcionar recursos para tornar mais direta a tarefa de comparação dos resultados com um software de simulação bem conhecido (Pspice). Adicionalmente, observa-se que os arquivos de simulação (net-lists) fornecidos pelas ferramentas para cada um dos conversores CC-CC analisados, foram criados empregando modelos idealizados convencionais de semicondutores e componentes (Mohan et alii, 1995; Rashid e Rashid, 2006).

Outro recurso disponível é o módulo "Design", como mostrado na Figura 6. Este módulo apresenta as equações básicas de projeto, considerando-se os parâmetros essenciais de operação (ganho estático, razão cíclica e modo de operação) e elementos circuitais (indutâncias, capacitâncias e resistências).

\section{METODOLOGIAS DE INTERAÇÃO}

Basicamente, a interação com as ferramentas de simulação propostas pode ser efetuada pelos usuários (estudantes)

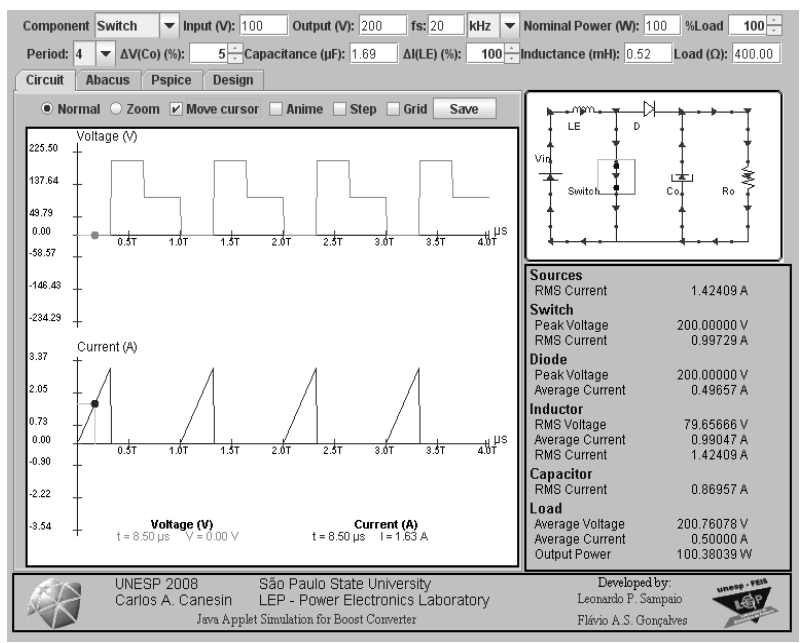

Figura 3: Conversor Boost, exemplos da forma de onda.

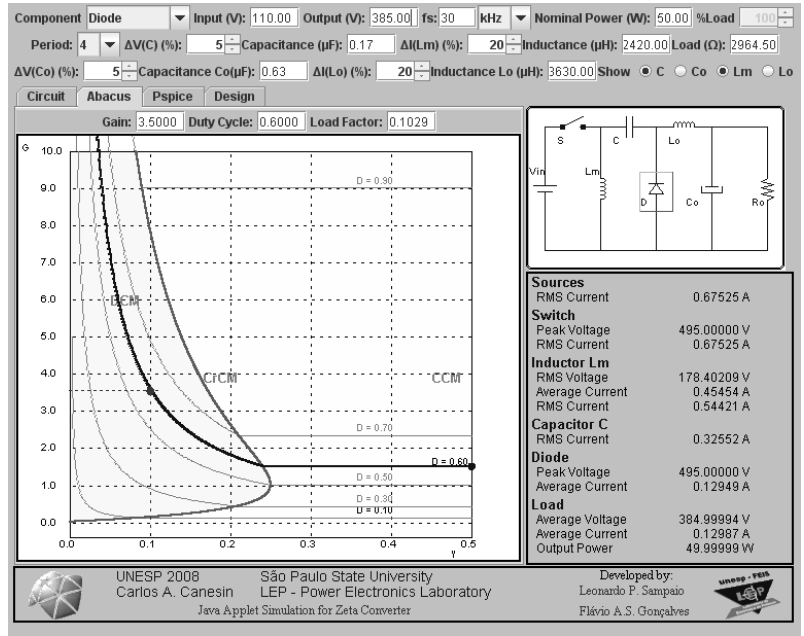

Figura 4: Conversor Zeta, exemplo do ábaco.

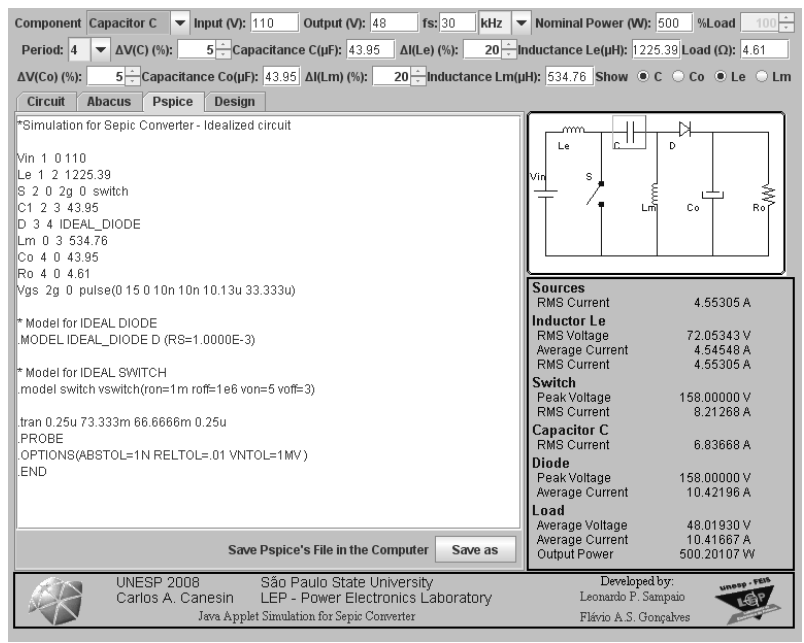

Figura 5: Conversor Sepic, exemplo do arquivo Pspice.

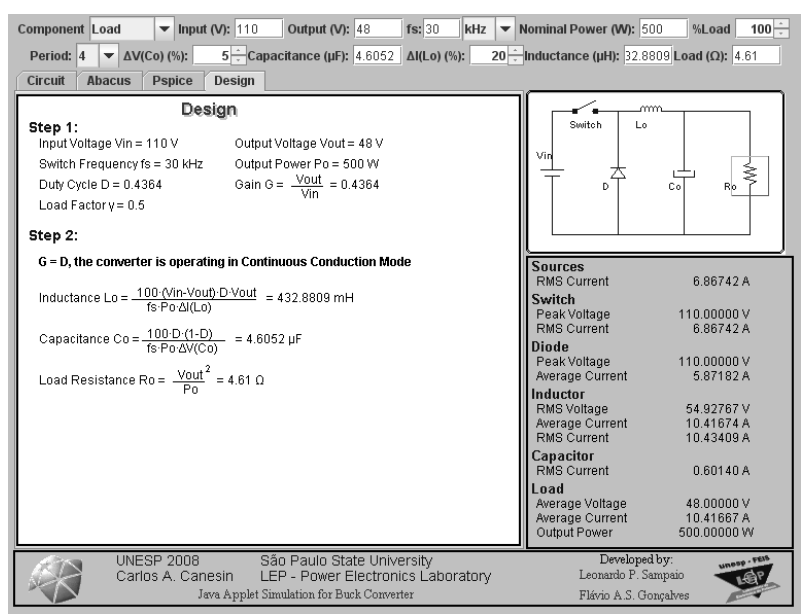

Figura 6: Conversor Buck, exemplo do módulo de projeto. 
usando o modo direto ou o modo gráfico.

As equações de projeto estão interligadas internamente, e cada mudança em quaisquer parâmetros resulta em um novo ponto de operação em regime permanente para o conversor relacionado. Além disso, sempre que a ferramenta é iniciada os parâmetros referentes a um projeto padrão são carregados.

No modo direto os parâmetros relacionados com o projeto do conversor podem ser inseridos simplesmente editando os valores do projeto padrão, tais como frequência de chaveamento, potência de saída, tensão de entrada/saída, ondulação da tensão, e assim por diante, apresentados em caixas de texto.

Portanto, a ferramenta de simulação permite que os estudantes explorem facilmente muitos aspectos da operação, apenas testando diferentes valores para um parâmetro específico.

Por exemplo, os estudantes podem avaliar os efeitos relacionados com a ondulação da tensão testando diferentes valores de capacitâncias, ou determinar o valor necessário do capacitor devido a uma especificação da ondulação da tensão. Assim, a mesma metodologia pode ser utilizada para explorar o efeito da indutância nas ondulações de corrente.

No modo gráfico os estudantes interagem indiretamente com as informações de projeto do conversor através da manipulação visual de componentes gráficos (no módulo do ábaco), efetuando as ações necessárias para alcançar os objetivos de projeto, tais como ganho estático, corrente normalizada e modo de condução.

A Figura 7 mostra um exemplo de interação no modo gráfico com a ferramenta de simulação do conversor Buck-boost, onde o ganho estático foi modificado pelo deslocamento do cursor vermelho ao longo da forma de onda de cor azul de 2,36 para 5,53, e, como consequência o modo de condução anteriormente MCC tornou-se MCD.

Além disso, enquanto o cursor vermelho se desloca no gráfico o parâmetro razão cíclica permanece constante.

De maneira análoga, a razão cíclica pode ser modificada pelo deslocamento do cursor azul ao longo do eixo vertical no gráfico do ábaco. Para realizar a atribuição, o ponteiro do mouse deve ser colocado em cima do cursor azul, e o estudante pode deslocar o cursor utilizando o botão direito do mouse, como mostra a Figura 8.

No exemplo mostrado na Figura 8, a razão cíclica aumentou de 0,2468 para 0,6212 e o ganho estático dobrou, enquanto a característica de carga normalizada permaneceu constante.

Assim, os resultados dos processos de simulação em regime permanente são visualmente apresentados ao estudante em tempo real, ilustrando conceitos importantes que são difíceis de demonstrar em uma sala de aula ou laboratório, sendo tal ferramenta de importância fundamental para este processo.

\section{SIMULAÇÃO E LABORATÓRIO INTE- RATIVOS}

Desde o início do curso os alunos podem interagir com a ferramenta proposta explorando os principais aspectos da operação de conversores, facilmente, sem sofrerem dos problemas convencionais enfrentados por inexperientes usando pacotes de simulação (problemas de convergência, especificação de tolerâncias, estabelecimento de modelos, etc...).

Na próxima fase os estudantes são solicitados a usarem os arquivos de configuração de simulação (net-lists) fornecidos pelas ferramentas propostas em um software de simulação conhecido, especificamente, Pspice, para estabelecer uma distinção entre ambas as abordagens computacionais. Assim, com a evolução do aprendizado/treinamento existe a tramitação gradual da ferramenta pré-configurada e idealizada para a ferramenta profissional, a medida que os estudantes se tornam capazes de efetuar modificações nos modelos ideais apresentados inicialmente, incorporando efeitos reais (resistências série, tensões em condução, capacitâncias e indutâncias, etc.), e podendo efetuar análises transitórias englobando respostas dinâmicas.

Os arquivos de configuração de simulação (net-lists) fornecidos estão baseados em uma biblioteca de modelos de componentes criada com as seguintes suposições: o capacitor de saída tem valor de capacitância suficiente para fornecer energia para a carga sem qualquer variação perceptível na sua tensão, a queda de tensão no diodo diretamente polarizado é nula, as perdas de comutação e condução nos interruptores e diodos são consideradas desprezíveis.

No entanto, estas suposições estão bem distantes da realidade, e as não-idealidades dos componentes reais podem produzir muitos efeitos sobre o funcionamento do conversor, desprezados nas abordagens simplificadas (ou ainda muito complexas de abordar completamente mesmo com modelos mais realistas (Pspice)) (Mohan et alii, 1995; Rashid e Rashid, 2006).

Desta forma, a avaliação prática de conversores de CC-CC, normalmente realizada nas aulas de laboratório, pode ser complementada com ferramentas de simulação interativas.

Consequentemente, os estudantes podem aprimorar os conceitos de Eletrônica de Potência criando uma analogia entre a teoria idealizada apresentada pelas ferramentas propostas e os resultados práticos obtidos nos laboratórios.

Normalmente, nas aulas de laboratório os conversores CC- 


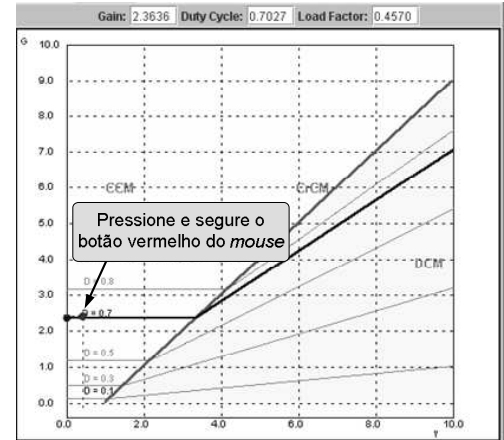

(a) Gráfico do ábaco considerando o conversor operando em MCC, e com ganho estático igual a 2,36.

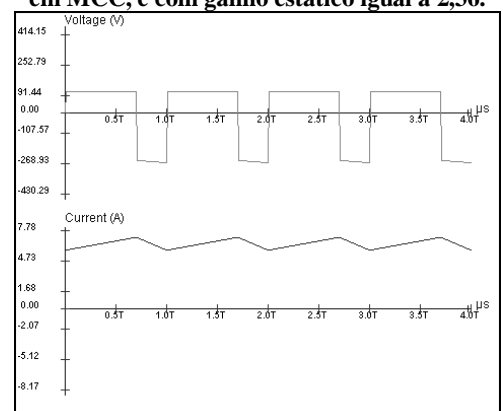

(c) Formas de onda no Indutor para o ganho de 2,36.

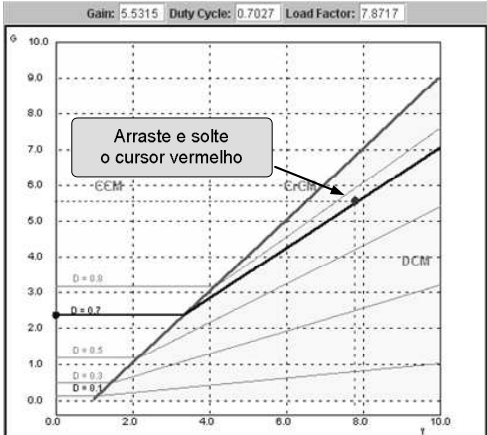

(b) Gráfico do ábaco considerando o conversor operando em MCD, e com ganho estático igual a 5,53.

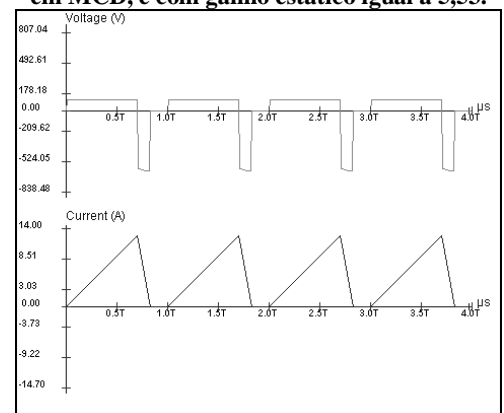

(d) Formas de onda no Indutor para o ganho de 5,53.

Figura 7: Ferramenta de simulação do conversor Buck-Boost mostrando um exemplo de interação no modo gráfico relacionado com a modificação do ganho estático, e, do modo de condução.

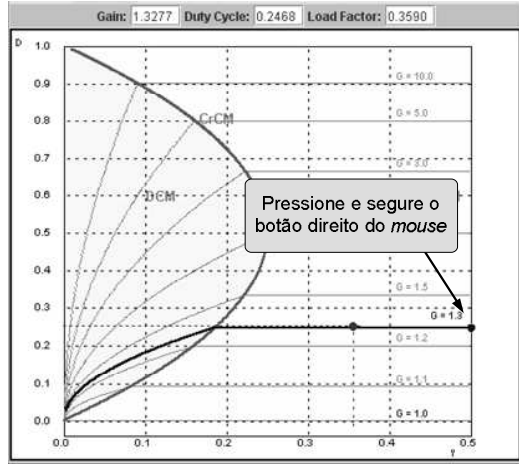

(a) Gráfico do ábaco com razão cíclica igual a 0,2468.

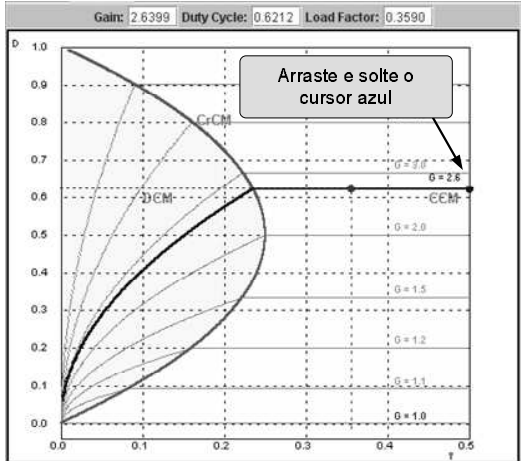

(b) Gráfico do ábaco com razão cíclica igual a 0,6212.

Figura 8: Ferramenta de simulação do conversor Boost mostrando um exemplo de interação no modo gráfico relacionado com a modificação da razão cíclica.

CC são implementados usando o controle com laço de realimentação único no modo tensão e modulação por largura de pulso (PWM - Pulse Width Modulation). Adicionalmente, um snubber dissipativo do tipo RC normalmente é inserido em paralelo com os terminais do interruptor principal para atenuar os esforços de tensão (efeitos de pico) durante a comutação para o bloqueio.

Assim, um exemplo de especificação para o projeto do conversor CC-CC Buck é apresentado na Tabela 1.
Inicialmente, os estudantes solucionam o problema dos requisitos de projeto usando a ferramenta de simulação proposta. Os valores dos componentes (indutância e capacitância), parâmetros operacionais e formas de onda são determinados instantaneamente pela ferramenta. Além disso, estudantes podem ver resumidamente como os componentes são calculados através do módulo "Design".

Considerando o exemplo de projeto proposto na Tabela 1, a ferramenta de simulação determina que o ganho estático e 
Tabela 1: Especificação de projeto para o conversor Buck.

\begin{tabular}{|l|c|}
\hline Parâmetros & Valores \\
\hline Tensão de Entrado (E) & $110 \mathrm{~V}$ \\
\hline Tensão de Saída (Vo) & $48 \mathrm{~V}$ \\
\hline Potência (Po) & $500 \mathrm{~W}$ \\
\hline Frequência (fs) & $30 \mathrm{kHz}$ \\
\hline $\begin{array}{l}\text { Ondulação de corrente na Indutância Lo } \\
\left(\mathrm{I}_{\text {Lo }}\right)\end{array}$ & $20 \%$ \\
\hline $\begin{array}{l}\text { Ondulação de tensão na Capacitância Co } \\
\left(\mathrm{V}_{C o}\right)\end{array}$ & $1 \%$ \\
\hline
\end{tabular}

Tabela 2: Comparação de resultados entre valor experimental, ferramenta de simulação e Pspice.

\begin{tabular}{|c|c|c|c|c|c|}
\hline Parâmetros & $\begin{array}{c}\text { Valor } \\
\text { Exp. }\end{array}$ & Applet & Pspice & $\begin{array}{c}\text { Erro }^{1} \\
(\mathbf{\%})\end{array}$ & $\begin{array}{c}\text { Erro }^{2} \\
(\boldsymbol{\%})\end{array}$ \\
\hline Chave S & & & & & \\
\hline $\begin{array}{c}\text { Tensão de Pico } \\
\text { (V) }\end{array}$ & 235,00 & 110,00 & 110,90 & 111,90 & 0,81 \\
\hline $\begin{array}{c}\text { Corrente Efi caz } \\
\text { (A) }\end{array}$ & 7,78 & 6,87 & 6,83 & 13,90 & 0,59 \\
\hline Diodo D & 195,00 & 110,00 & 110,00 & 77,27 & 0,00 \\
\hline $\begin{array}{c}\text { Tensão de Pico } \\
\text { (V) }\end{array}$ & 5,47 & 5,87 & 5,82 & 6,00 & 0,86 \\
\hline $\begin{array}{c}\text { Corrente Média } \\
\text { (A) }\end{array}$ & 10,70 & 10,42 & 10,32 & 3,68 & 0,97 \\
\hline $\begin{array}{c}\text { Indutor Lo } \\
\text { Tensão Efi caz (V) }\end{array}$ & 51,80 & 54,20 & 55,00 & 5,82 & 1,45 \\
\hline $\begin{array}{c}\text { Corrente Média } \\
\text { (A) }\end{array}$ & 10,70 & 10,42 & 10,31 & 2,52 & 1,07 \\
\hline $\begin{array}{c}\text { Corrente Efi caz } \\
\text { (A) }\end{array}$ & 10,72 & 10,43 & 10,33 & 3,78 & 0,97 \\
\hline $\begin{array}{c}\text { Carga Ro } \\
\text { Tensão Média (V) }\end{array}$ & 50,83 & 48,00 & 47,53 & 6,94 & 0,99 \\
\hline $\begin{array}{c}\text { Corrente Média } \\
\text { (A) }\end{array}$ & 10,57 & & & \\
\hline
\end{tabular}

1- Erro entre o valor experimental e o Pspice.

2- Erro entre a ferramenta de simulação e o Pspice.

3- Mosfet IRF740 - RC Snubber $(\mathrm{R}=47 \Omega$ e C $=2,2 \mathrm{nF})$.

a razão cíclica devem possuir o mesmo valor, 0,4364 , refletindo no modo de condução MCC. Além disso, para a conformidade com as especificações de ondulação de tensão e de corrente estabelecidas, a ferramenta determina que os valores da indutância (Lo) e da capacitância (Co) são respectivamente $437 \mu \mathrm{H}$ e $330 \mu \mathrm{F}$.
$\mathrm{Na}$ fase seguinte os estudantes são motivados a confrontar os resultados com outro pacote de simulação (Pspice) e com resultados experimentais.

Assim, o conversor CC-CC Buck do exemplo de projeto é criado e testado experimentalmente nas aulas de laboratórios para fornecer recursos para a comparação entre as metodologias.

A Figura 9 e a Tabela 2 mostram alguns resultados obtidos nas aulas de laboratório para as três abordagens (ferramenta de simulação proposta, Pspice e resultados experimentais).

A comparação das formas de onda de comutação (tensão e corrente) no interruptor provenientes da ferramenta proposta e do Pspice com aquelas obtidas na avaliação experimental indicam claramente os efeitos não abrangidos pela teoria idealizada, como é mostrado na Figura 9.

Os coeficientes de erros para as três abordagens são comparados na Tabela 2, onde se comprova que exceto para o valor de pico de tensão os valores de erro entre as ferramentas de simulação e os resultados experimentais permaneceram abaixo de $1,5 \%$.

Estes resultados comparativos dão suporte à comprovação de que a teoria idealizada apresentada nas salas de aula é eficaz, e, capaz de representar a grande maioria dos comportamentos dos conversores $\mathrm{CC}-\mathrm{CC}$ em regime permanente.

Por último, a utilização das ferramentas propostas durante as experiências de laboratórios convencionais propicia aos estudantes condições para realização de comparações em temporeal entre os resultados obtidos nas experiências de laboratórios e os conceitos teóricos desenvolvidos nas classes. A metodologia resultante é muito eficiente para o ensino, onde os alunos estão ativamente envolvidos, estando imersos em um sistema de aprendizagem interativo e atraente.

\section{SOFTWARE EDUCACIONAL}

As ferramentas interativas podem ser facilmente incorporadas em softwares educacionais porque foram totalmente desenvolvidas usando as vantagens da linguagem Java (arquitetura neutra propiciando independência de plataforma, fácil incorporação ao ambiente web, desenvolvimento rápido, portabilidade e robustez).

Um software educacional foi desenvolvido usando tanto uma plataforma aberta baseada em tecnologias da internet quanto uma plataforma autônoma (stand-alone) para sistema operacional Windows, proporcionando um mecanismo eficaz para divulgação do curso e dos aplicativos de simulação no formato eletrônico para os estudantes e professores. 


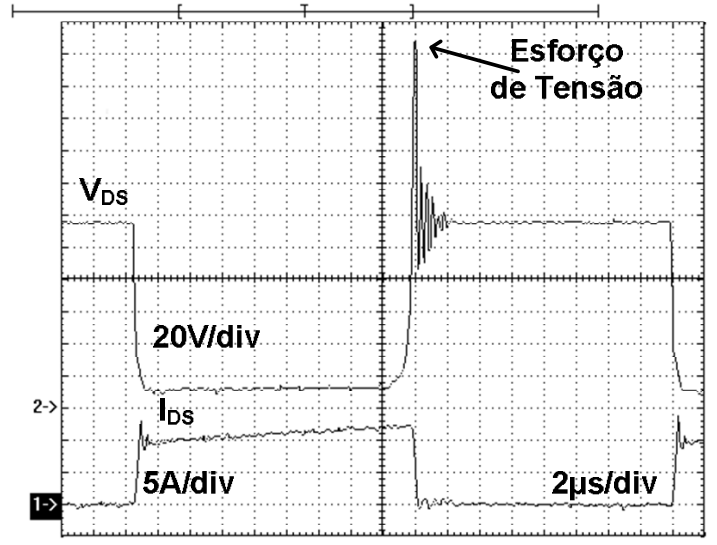

(a) Experimental.

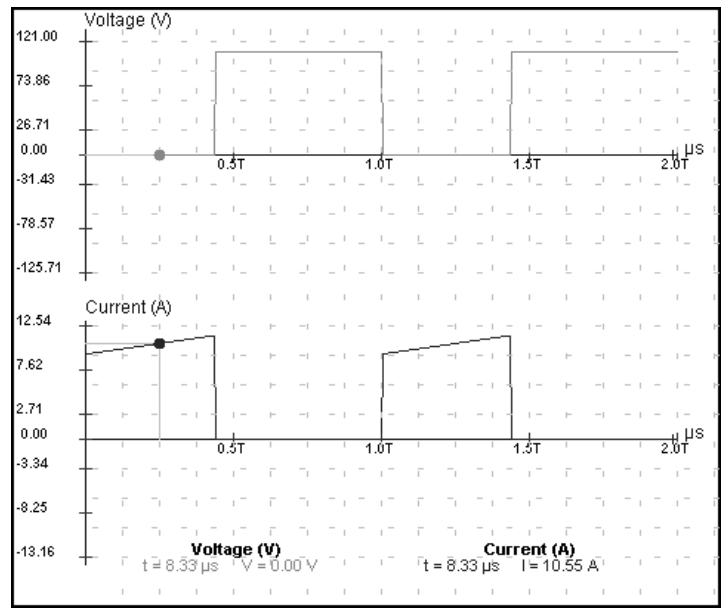

(b) Ferramenta de simulação proposta.

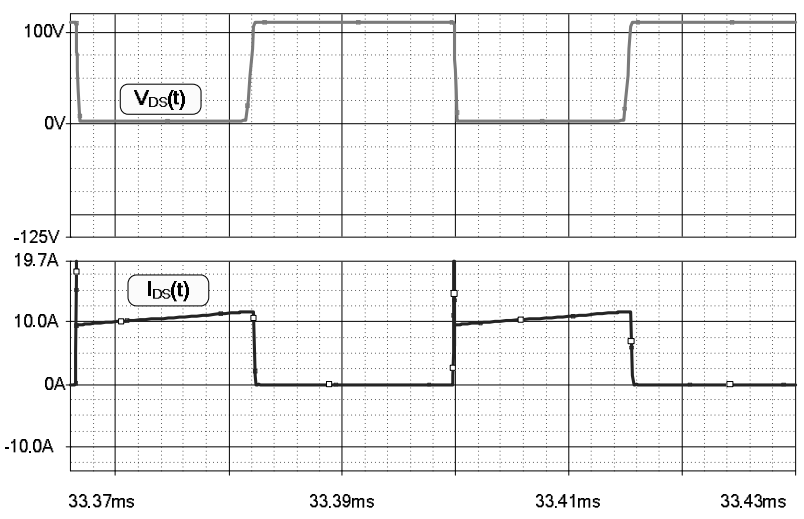

(c) Software de simulação Pspice.

Figura 9: Formas de onda da comutação no interruptor do conversor Buck considerando três metodologias.

Embora este artigo tenha centrado foco apenas no tópico conversores CC-CC não-isolados em regime permanente, várias ferramentas de simulações foram desenvolvidas cobrindo ou-

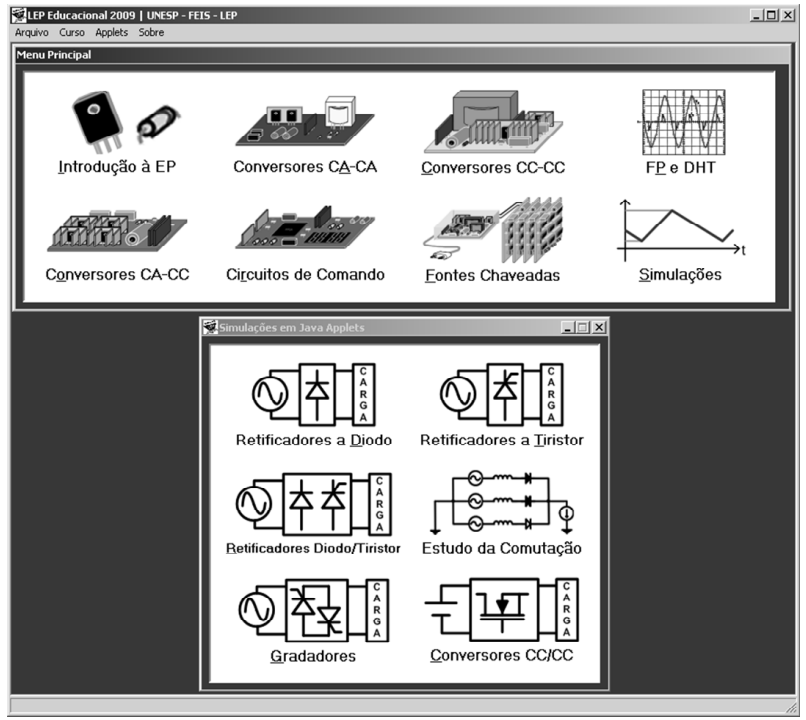

Figura 10: Software Educacional para auxílio no curso de Eletrônica de Potência.

tros tópicos relacionados com a disciplina de Eletrônica de Potência, tais como, retificadores CA-CC e conversores CACA.

A plataforma completa para auxiliar o ensino de Eletrônica de Potência inclui em um software educacional todas as ferramentas interativas desenvolvidas até o momento.

Além das ferramentas de simulações, a plataforma fornece todo o material didático apresentado nas salas de aula, incluindo apresentações, exercícios e problemas propostos. $\mathrm{O}$ material didático cobre tópicos como retificadores $\mathrm{CA}-\mathrm{CC}$, conversores CA-CA, conversores CC-CC, fontes de alimentação chaveadas, teoria básica para fator de potência (F.P.), distorção harmônica total (D.H.T.) e algumas normas internacionais de regulação (IEC6 1000-3-2, 2002; IEC 61000-34, 2002).

Além disso, o acesso às ferramentas de simulação durante a utilização do aplicativo pelos professores e alunos é imediato, pois as mesmas estão inseridas em todos os textos dos tópicos relacionados com a sua utilização.

A versão stand-alone em Delphi do software educacional proposto é mostrada na Figura 10. A escolha da plataforma Delphi para o desenvolvimento do módulo gerencial da versão fechada foi baseada principalmente em função do domínio da mesma pelos autores e na possibilidade de utilização de orientação a objeto.

As novas ferramentas de simulação estão disponíveis para utilização gratuita, tanto para professores como para os estudantes, no site do Laboratório de Eletrônica de Po- 
tência na UNESP de Ilha Solteira, através do endereço: http://www.dee.feis.unesp.br/docentes/flavio/lepjava.

\section{CONCLUSÕES}

Este artigo apresentou ferramentas interativas de simulações para conversores CC-CC, propostas para servir de auxílio ao ensino e projeto inicial destas estruturas nas aulas e experiências de laboratório no curso de Eletrônica de Potência.

As ferramentas educacionais propostas permitem que os alunos possam adquirir treinamento em conversores CC-CC não-isolados, sem requerer o prévio conhecimento dos circuitos, e tampouco o uso de softwares de simulação sofisticados que envolvem elevado custo e um tempo longo para a aprendizagem da sua utilização.

No entanto, os pacotes de simulação de circuitos são instrumentos essenciais em cursos de engenharia e altamente recomendados para a realização de análises complementares e avançadas das topologias, uma vez que são capazes de fornecer modelos complexos e com comportamento muito próximo da realidade.

Assim, concluído o processo inicial de aprendizado pelos alunos, onde os principais conceitos de operação dos conversores foram adquiridos, as ferramentas educacionais propostas passam para um papel secundário, apoiando os alunos na melhoraria das suas habilidades nos pacotes de simulação. Ajudando inicialmente no fornecimento dos arquivos de configuração de simulação (net-lists) de conversores CC-CC, e depois como fonte de dados para o desenvolvimento de uma comparação entre ambas as abordagens computacionais.

Além disso, a utilização das ferramentas propostas durante as experiências de laboratório proporciona um ambiente onde os estudantes podem realizar análises comparativas em tempo-real considerando os resultados visualizados nas experiências de laboratório e os conceitos teóricos desenvolvidos nas salas de aula.

A metodologia de ensino resultante é muito eficiente, uma vez que os alunos estão ativamente envolvidos num ambiente que permite uma aprendizagem interativa e atraente.

Finalmente, à medida que a metodologia proposta foi incluída nas salas de aula tradicionais, constatou-se uma elevação importante no interesse dos alunos em aprender o conteúdo do curso de Eletrônica de Potência, resultando em uma importante melhoria da aprendizagem.

\section{AGRADECIMENTOS}

Agradecemos o suporte financeiro do CNPq (Conselho Nacional de Tecnologia e Desenvolvimento Científico) no desenvolvimento desta pesquisa.

\section{REFERÊNCIAS}

Agelidis, V. G. (2005). The future of power electronics/power engineering education: challenges and opportunities, in Proc. Of IEEE Power Electronics Education Workshop, Recife, Brasil, vol. 1, pp. 1-8.

Bauer, P.; Feadk, V. e Hajek, V. (2008). Survey of Distance Laboratories in Power Electronics, in Proc. Of IEEE Power Electronics Specialists Conference, Rhodes, Grécia, vol. 1, pp. 430-436.

Barbi, I. e Martins, D. C. (2000). Eletrônica de Potência: Conversores CC-CC Básicos Não Isolados, $3^{a}$ Edição, Florianópolis.

Bartal, P.; Bauer, P.; Hamar, J.; Jardan, R. K.; Korondi, P.; Nagy, I.; Sütö, Z.; Zabán, K.; Funato, H. e Ogasawara, S. (2005). Multimedia Course for Power Electronics, Nonlinear Dynamics and Motion Control, in Proc. Of IEEE Power Electronics Education Workshop, Recife, Brasil, vol. 1, pp. 74-80.

Canesin, C. A.; Gonçalves, F. A. S. e Sampaio, L. P. (2008). Simulation tools for power electronics courses, based in Java Technologies, Eletrônica de Potência, vol. 13, no. 2, pp. 91-98.

Donoso-Garcia, P. F.; Cortizo, P. C. e Morais L. M. F. (2008). Ensino orientado ao projeto: uma experiência para o ensino de eletrônica nas disciplinas de laboratório de eletrônica e eletrônica de potência, Eletrônica de Potência, vol. 13, no. 2, pp. 109-116.

Drofenik, U. e Kolar, J. W. (2002). Interactive Power Electronics Seminar (iPES)-a Web-Based Introductory Power Electronics Course Employing Java Applets, in Proc. Of IEEE Power Electronics Specialists Conference, Cairns, Austrália, vol. 2 , pp. 443-448.

Erickson, R. W. e Maksimovic, D. (2002). Fundamentals of Power Electronics, Kluwer Academic Publisher, $2^{\text {nd }}$ Edition, USA.

Garcia, F. S.; Ferreira, A. A. e Pomilio, J. A. (2008). Plataforma de ensino de eletrônica de potência versátil e de baixo custo, Eletrônica de Potência, vol. 13, no. 2, pp. 85-90.

Gonçalves, F. A. S. e Canesin, C. A. (2002). Java applets para um software educacional distribuído em eletrônica 
de potência, SBA Controle \& Automação, vol. 13, no. 3, pp. 314-326.

Gonçalves, F. A. S. e Canesin, C. A. (2005). Educational Java applets get in power electronics labs, in Proc. Of IEEE Power Electronics Education Workshop, Recife, Brasil, vol. 1, pp. 34-39.

Harb, S.; Kalaldiah, K.; Harb, A. e Batarseh, I. (2005). Interactive JAVA Applets for Power Electronics ELearning, in Proc. Of IEEE Power Electronics Education Workshop, Recife, Brasil, vol. 1, pp. 26-33.

Hwang, G. (1998). A Tutoring Strategy Supporting System For Distance Learning On Computer Networks, IEEE Transactions on Education, vol. 41, no. 4, pp. 343-343.

International Electrotechnical Commission (2002). IEC 61000-3-2: "Electromagnetic Compatibility (EMC) Part 3: Limits - Section 2: Limits for Harmonic Current Emissions (Equipment input current $<16 \mathrm{~A} \mathrm{per}$ phase)", $2^{\text {nd }}$ Edition.

International Electrotechnical Commission (2002). IEC 61000-3-4: "Limitation of emission of harmonic currents in low-voltage power supply systems for equipment with rated current greater than 16A", $2^{\text {nd }}$ Edition.

Mohan, N.; Undeland, T. M. e Robbins, W. P. (1995). Power Electronics: converters, applications, and design, John Wiley \& Sons, $2^{\text {nd }}$ Edition, New York, USA.

Oliveira Júnior, A. S. e Silva, E. R. C. (2004). Software Educacional em C++ para Ensino e Simulação de Inversores PWM em Eletrônica de Potência, Anais do Congresso Brasileiro de Automática, Gramado, Brasil.

Pleskacz, W. A.; Stopjaková, V.; Borejko, T.; Jutman, A. e Walkanis, A. (2008). DefSim: A Remote Laboratory for Studying Physical Defects in CMOS Digital Circuits, IEEE Transactions on Industrial Electronics, vol. 55 , no. 6, pp. 2405-2415.

Ramos, P. M.; Silva, M. F.; Martins, R. C. e Cruz Serra, A. M. (2006). Simulation and Experimental Results of Multiharmonic Least-Squares Fitting Algorithms Applied to Periodic Signals, IEEE Transactions on Instrumentation and Measurement, vol. 55, no. 2, pp. 646-651.

Rashid, M. H. e Rashid, H. M. (2006). SPICE for Power Electronics and Electric Power, CRC Press, $2^{\text {nd }}$ Edition, USA.

Silva, E. M. e Cunha, J. P. V. S. (2006). SCILAB, SCICOS e RLTOOL: Softwares Livres no Ensino de Engenharia Elétrica, Anais do Congresso Brasileiro de Automática, Salvador, Brasil, pp. 1620-1625.
Wu, M.; She, J.; Zeng, G. e Ohyama, Y. (2008). InternetBased Teaching and Experiment System for Control Engineering Course, IEEE Transactions on Industrial Electronics, vol. 55, no. 6, pp. 2386-2396. 